LA WAENCE LIWEAMCAE NATIONAL LABOAATOAY

\title{
Axial Electron Heat Loss From Mirror Devices Revisited
}

D.D. Ryutov

August 17, 2004

5th International Conference on Open Magnetic Systems for Plasma Confinement

Novosibirsk, Russia

July 5,2004 


\section{DISCLAIMER}

This document was prepared as an account of work sponsored by an agency of the United States Government. Neither the United States Government nor the University of California nor any of their employees, makes any warranty, express or implied, or assumes any legal liability or responsibility for the accuracy, completeness, or usefulness of any information, apparatus, product, or process disclosed, or represents that its use would not infringe privately owned rights. Reference herein to any specific commercial product, process, or service by trade name, trademark, manufacturer, or otherwise, does not necessarily constitute or imply its endorsement, recommendation, or favoring by the United States Government or the University of California. The views and opinions of authors expressed herein do not necessarily state or reflect those of the United States Government or the University of California, and shall not be used for advertising or product endorsement purposes.

This work was performed under the auspices of the U.S. Department of Energy by the University of California, Lawrence Livermore National Laboratory under Contract No. W-7405-Eng-48. 


\title{
AXIAL ELECTRON HEAT LOSS FROM MIRROR DEVICES REVISITED
}

\author{
D.D. Ryutov \\ Lawrence Livermore National Laboratory, Livermore, CA 94551, USA
}

An issue of the axial electron heat loss is of a significant importance for mirror-based fusion devices. This problem has been considered in a number of publications but it is still shrouded in misconceptions. In this paper we revisit it once again. We discuss the following issues: 1) Formation of the electron distribution function in the end tank at large expansion ratios; 2) The secondary emission from the end plates and the ways of suppressing it (if needed); 3) Ionization and charge exchange in the presence of neutrals in the end tanks; 4) Instabilities caused by the peculiar shape of the electron distribution function and their possible impact on the electron heat losses; 5) Electron heat losses in the pulsed mode of operation of mirror devices.

\section{INTRODUCTION}

In the open confinement systems, by their very definition, the region of a hot fusion plasma is directly connected along the field lines with a cold material walls. This fact is sometimes considered as leading to an inevitable failure of the whole concept of the mirror confinement, because of very large electron heat losses along the field lines. On the other hand, those involved in mirror research, know that there is no fundamental limit on the electron temperature imposed by the parallel heat loss, unless there is strong secondary electron emission from the end walls. In this paper, the author presents a brief summary of the earlier results and some new estimates related to the ways by which the detrimental effect of the secondary emission can be substantially reduced or eliminated.

The discussion is limited to a qualitative level and order-of-magnitude estimates. Earlier studies of the axial losses are summarized in Refs. 1-4.

\section{GENERAL CHARACTERIZATION OF THE PLASMA BEYOND THE MIRROR THROAT}

We concentrate on the situation where the mean free path of electrons leaving the device is much greater than the distance between the outermost mirror and the end wall. In other words, the hot electrons are in a deeply kinetic regime, at least in the end tank. In the absence of the secondary emission, electron confinement under such circumstances has been considered in a number of papers, in particular, in Refs. 4 and 5. The opposite limit can be treated within the two-fluid transport equations [6].

If there is no secondary emission, and the axial losses are dominant over the cross-field losses for both electrons and ions, the quasineitrality constraint means that one electron is lost per one ion along the field lines. This is reached by the formation of an electrostatic potential barrier that holds the electron loss equal to the ion loss; as the electron collision frequency is much higher than the ion collision frequency, one can then assume that the electrons that are leaving the device have a Maxwellian distribution, with the temperature equal to that of the electrons in the confinement region. [Some deviations from the Maxwellian may occur at very large mean free paths [2]]. According to what has just been said, the electrons, in order to leave the confinement device, have to overcome a high potential barrier, and the average energy $\bar{W}_{e}$ of the electrons leaving the confinement region is substantially higher than $T_{e}$,

$$
\bar{W}_{e}=A_{e} T_{e} \text {, }
$$

with the coefficient $A_{e}=5-8$, depending on the details of the confinement scheme.

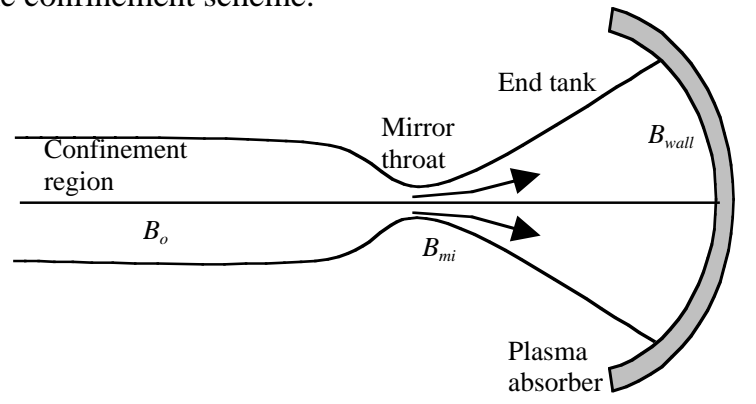

Fig. 1. Schematic of the end section. Arrows show the plasma flowing out of the confinement region and expanding towards the end wall (plasma absorber).

As the average energy of the ions leaving the device is only by a factor of $\sim 2$ higher than their "temperature," the electrons in a mirror device with a classical confinement will be colder than the ions (unless there is a separate electron heating source). Maybe, just this conclusion is the basis for a legend about the "dead cold" electrons in mirror devices. However, the fact that electrons are colder than the ions does not by itself make the mirror reactor impossible: one has just to take into account the ion energy loss by the drag against electrons 
which somewhat reduces the fusion gain (for more detail see, e.g., Ref. [3]).

Fig. 1 depicts the schematic of the end section of some generic mirror device. In order to maintain a manageable heat load on the plasma absorber, the expansion ratio of the magnetic field in the end tank,

$$
K_{\text {wall }} \equiv \frac{B_{\text {mir }}}{B_{\text {wall }}}
$$

has to be very large, in the range between 300 and 3000 .

The ion flow just beyond the mirror throat has a velocity somewhat exceeding the ion thermal velocity, because of the potential drop in the mirror region. The flow continues to accelerate under the action of the ambipolar potential in the end tank. However, this acceleration is not very substantial, since the electron temperature is a few times lower than the ion temperature. Accordingly, in the first rough approximation, one can consider the flow velocity $u$ just as a constant. Then, the continuity equation shows that the plasma density in the expander deceases just as the magnetic field, i.e.,

$$
\frac{n}{n_{m i r}}=\frac{B}{B_{m i r}}=\frac{1}{K},
$$

where we use a notation $K$ for the running expansion ratio.

Table I Characteristic parameters for the "canonical" mirror and the gas-dynamic trap*.

\begin{tabular}{|l|c|c|c|c|c|}
\hline & $\begin{array}{c}a, \\
\mathrm{~m}\end{array}$ & $\begin{array}{c}R \\
\left(K_{\text {wall }}\right)\end{array}$ & $\begin{array}{c}T_{e}\left(T_{i}\right) \\
\mathrm{keV}\end{array}$ & $\begin{array}{c}n^{(\text {wall })} \\
\mathrm{cm}^{-3}\end{array}$ & $\begin{array}{c}q^{(\text {wall })} \\
\mathrm{MW} / \mathrm{m}^{2}\end{array}$ \\
\hline $\begin{array}{l}\text { "Canonical" } \\
\text { mirror }\end{array}$ & 1 & $\begin{array}{c}3 \\
(300)\end{array}$ & $\begin{array}{c}30 \\
(100)\end{array}$ & $1.4 \times 10^{6}$ & 0.48 \\
\hline GDT & 0.6 & $\begin{array}{c}20 \\
(3000)\end{array}$ & $6(6)$ & $9 \times 10^{7}$ & 0.89 \\
\hline
\end{tabular}

*In both cases we assume that fusion power $P$ is $1 \mathrm{GW}, Q=10, A_{e}=6$, $A_{i}=2, u=2 \sqrt{2 T_{i} / m_{i}}$, with the ion mass being 2.5 proton mass.

The energy flux at the end wall can be evaluated as

$$
q^{(\text {wall })}=\frac{P R(0.2+1 / Q)}{2 \pi a^{2} K_{\text {wall }}},
$$

where $P$ is the fusion power, $R$ is the mirror ratio, $Q$ is the fusion gain (fusion power/power in), and $a$ is the plasma radius in the confinement region; the first term in the brackets accounts for the alpha heating; the factor " 2 " in the denominator accounts for two ends. On the other hand, one has

$$
q^{(\text {wall })} \approx n^{(\text {wall })} u\left(A_{e} T_{e}+A_{i} T_{i}\right) .
$$

Equating the two, one finds the following expression for the plasma density near the end plate:

$$
n^{(\text {wall })}=\frac{P R}{2 \pi a^{2} Q K u\left(A_{e} T_{e}+A_{i} T_{i}\right)} .
$$

Numerical values of various parameters are presented in Table I, both for a "canonical" mirror, by which we mean a system with a particle mean free path much exceeding the length of the confinement region [7], and a higherdensity, short mean free path device called "gas dynamic trap," or GDT [8].

\section{SUMMARY OF THE PREVIOUS WORK}

The potential distribution in the end tank was studied in Ref. [5]; some further details have been added in Ref. [4]. The electrostatic potential has to maintain a quasineutrality condition over the end tank.

There is, roughly, a thousand-fold decrease of the plasma density between the mirror throat and the end wall. This means that the majority of the electrons are reflected from the electrostatic potential barrier and return back to the confinement region. One might, therefore, expect that the electron distribution in the end tank is close to the Maxwell-Boltzmann distribution with the temperature roughly equal to the electron temperature in the confinement region. Following this line, one would obtain the following relation between the potential and the expansion ratio:

$$
\exp \left[-e\left(\varphi_{\text {mir }}-\varphi\right) / T_{e}\right]=1 / K
$$

Note that collisions are important despite our assumption that the length of the expander $L_{\text {exp }}$ is much less than the electron collision mean-free path. What matters in the context of the expander distribution, is the collision frequency. If the duration of the experiment is much longer than the electron collision time in the expander, then the distribution function is affected by collisions [5].

In the immediate vicinity of the wall there is a Debye sheath whose magnitude $\Delta \varphi_{D}<0$ is such as to maintain the electron and ion fluxes to the wall equal. The electron particle flux in the mirror is determined by the following expression:

$$
n_{\text {mir }} \mathrm{v}_{T e} \exp \left[-e\left(\varphi_{\text {mir }}-\varphi_{\text {wall }}-\Delta \varphi_{D}\right) / T_{e}\right],
$$

where $\varphi_{\text {wall }}$ is the plasma potential immediately outside the Debye sheath. We have used the fact that the electron mean free path is much longer than $L_{\text {exp }}$. By comparing Eqs. (7) and (8), one sees that the electron flux in the mirror throat is

$$
\frac{n_{m i r} \mathrm{v}_{T e}}{K_{\text {wall }}} \exp \left(e \Delta \varphi_{D} / T_{e}\right) .
$$

The ion flux in the mirror throat is $n_{m i r} u$. One sees that, at

$$
K_{\text {wall }} \gg \frac{\mathrm{v}_{T e}}{u} \sim \sqrt{\frac{m_{i}}{m_{e}}}
$$

the electron loss formally becomes much less than the ion loss, in an obvious contradiction to the charge neutrality of the confined plasma. [At $K_{\text {wall }}<\sqrt{m_{i} / m_{e}}$ the electron flux is adjusted to the ion flux by the potential jump $\Delta \varphi_{D}<0$ in the Debye sheath, Fig. 2, the curve for $\left.K_{m i r}=10\right]$.

This shows that, at a very large expansion ratios, the potential difference between the wall and the mirror throat cannot increase substantially over the $\left(T_{e} / e\right) \ln \sqrt{m_{i} / m_{e}}$. 
If $K_{\text {wall }}$ exceeds $\sqrt{m_{i} / m_{e}}$, then the potential variation in the zone where

$$
\sqrt{m_{i} / m_{e}}<K<K_{\text {wall }}
$$

becomes small compared to $T / e$. This means that the distribution function for $K$ satisfying Eq. (10) significantly differs from the Maxwellian with the temperature $T_{e}$.

The distribution function in this zone is formed by the scattering off the transiting electron stream; scattered electrons form a quasi-spherical distribution function. The influx of particles to this distribution is balanced by the particle loss to the wall. The characteristic velocity $\hat{v}$ of the spherical part of the distribution can be found from the observation that the velocity-space density of the transiting particles at $\mathrm{v}<\hat{\mathrm{v}}$ is not much different from that of trapped particles, as othervise the scattering over a small angle $\sim 1 / K^{1 / 2}$ would smoothen the difference. This yields the following estimate for the density of trapped electrons:

$$
n_{m i r}\left(\frac{\hat{\mathrm{v}}}{\mathrm{v}_{T e}}\right)^{3} \frac{u}{\mathrm{v}_{T e}} .
$$

Equating this to the ion density, i.e., to $n_{m i r} / K$, one finds the characteristic velocity $\hat{\mathrm{V}}$ and the "temperature" $\hat{T}_{e} \sim \mathrm{m}_{\mathrm{e}} \hat{\mathrm{v}}^{2} / 2$ of the trapped electrons:

$$
\begin{aligned}
& \hat{\mathrm{v}} \sim \mathrm{v}_{T e}\left(\frac{\mathrm{v}_{T e}}{K u}\right)^{1 / 3} \sim \frac{\mathrm{v}_{T e}}{K^{1 / 3}}\left(\frac{m_{i}}{m_{e}}\right)^{1 / 6}, \\
& \hat{T}_{e} \sim T_{e}\left(\frac{\mathrm{v}_{T e}}{K u}\right)^{2 / 3} \sim \frac{T_{e}}{K^{2 / 3}}\left(\frac{m_{i}}{m_{e}}\right)^{1 / 3} .
\end{aligned}
$$

This estimate works for $K>\left(m_{t} / m_{e}\right)^{1 / 2}$. Note that the density of trapped electrons, $n_{\text {mir }} / K$, is much higher than the density of the transiting electrons (the latter is $\left.\left(n_{\text {mir }} / K\right)\left(m_{e} / m_{i}\right)^{1 / 2}\right)$.

The potential difference between the wall and the observation point is determined just from the condition that the trapped electrons are confined,

$$
e\left(\varphi-\varphi_{\text {wall }}-\Delta \varphi_{D}\right) \sim \hat{T}_{e} .
$$

The potential drop in the Debye sheath can be evaluated as

$$
-e \Delta \varphi_{D} \sim \frac{T_{e}}{K_{\text {wall }}^{2 / 3}}\left(\frac{m_{i}}{m_{e}}\right)^{1 / 3} .
$$

Table II summarizes parameters of the near-wall plasma (its density was presented in the second-to-last column of Table 1). Note that the electron temperature of trapped electrons remains quite high, although lower than the electron temperature in the confinement region. Because of a high temperature and low density of the near-wall plasma, collision times in it are quite long, approaching $\sim 2$ hours in the case of a canonical mirror. This means that the distribution function near the walls can be very susceptible to all kinds of perturbations.

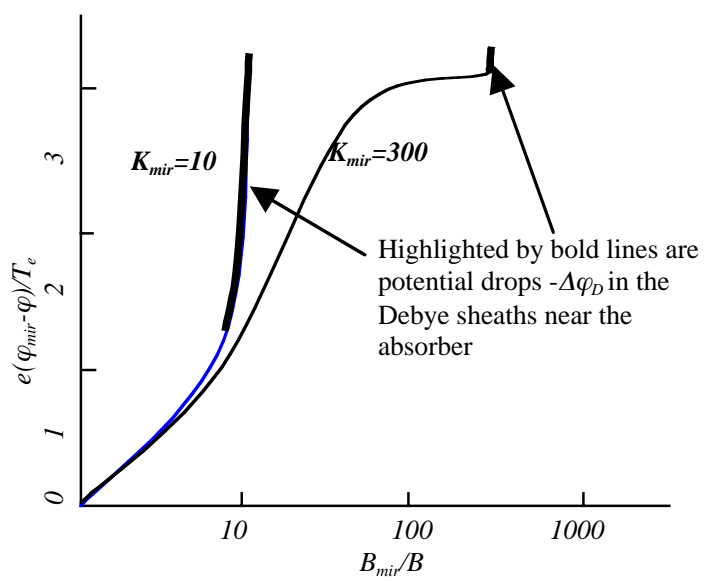

Fig. 2 Potential distribution for the absorber situated at $K=10$, and $K=300$. Note a flattening of the potential in the zone of a weak magnetic field in the second case. The plasma potential at the bottom of thick lines is what is called $\varphi_{\text {wall }}$ in the body of the paper.

Table II. Plasma parameters near the absorbing wall*.

\begin{tabular}{|l|c|c|c|c|c|}
\hline & $B_{0}, T$ & $\begin{array}{c}B_{\text {wall }} \\
T\end{array}$ & $\begin{array}{c}\hat{T}_{e}^{(\text {wall })} \\
\mathrm{keV}\end{array}$ & $\left(\frac{\omega_{c e}}{\omega_{p e}}\right)^{\text {(wall) }}$ & $\begin{array}{c}\hat{\tau}_{e i}^{(\text {wall })} \\
\mathrm{s}\end{array}$ \\
\hline $\begin{array}{l}\text { "Canonical" } \\
\text { mirror* }\end{array}$ & 5 & 0.05 & 3.1 & 130 & $6 \cdot 10^{3}$ \\
\hline GDT & 1.5 & 0.01 & 0.48 & 2.5 & 5 \\
\hline
\end{tabular}

* All the input parameters are the same as in Table I; $B_{0}$ is the magnetic field in the confinemnt region; $B_{\text {wall }}=B_{0} R / K_{\text {wall }}$.

\section{SUPPRESSION OF THE SECONDARY EMISSION}

\section{IV.A. A detrimental effect of large secondary emission}

Electrons can be liberated from the end plate by a variety of mechanisms. Here, for brevity, we shall assume that the only process that leads to the emission of electrons from the end plate is the secondary electronelectron emission. We characterize it by the secondary emission coefficient $\eta$. If other mechanisms are present, one should just renormalize $\eta$ appropriately.

The presence of the secondary emission leads to an increase of the electron heat flux from the plasma. Indeed, in every event of the secondary emission, the primary electron that hits the wall with the energy $\sim T_{e}$ (many kiloelectron-volts) is replaced by a secondary electron that leaves the wall with the typical energy of only a few electron-volts. Accordingly, the pre-existing electron heat flux $q_{0}$ increases, roughly, by a factor of $\left(1+\eta / A_{e}\right)$ which is not much greater than 1. (Cf. a similar analysis in Ref. [9].) 
The situation changes dramatically when the coefficient $\eta$ becomes close to 1 or exceeds 1 . In the latter case, potentially, the heat loss through the electron channel may occur even if the ion loss is just zero, as every hot electron leaving the device would be replaced by a secondary electron, thereby eliminating the quasineutrality constraint that leads to a relation between the particle loss and the electron heat loss used in the previous discussion. The accumulation of the cold secondary electrons would gradually reduce the potential drop in the end tank and thereby lead to an un-inhibited electron heat loss. Clearly, this situation is undesirable and the ways of avoiding this scenario have to be considered. The conclusion of Ref. [5] on the same matter was overly optimistic.

The most desirable solution would be just finding the materials for which $\eta$ is less than, say, 0.5. However, it is at present unclear whether such materials can actually be found, especially given that the surface layer of the end plate will be saturated with hydrogen and the end wall will be bombarded with electrons and ions having energies extending to many kilo-electron-volts.

Note that at $\eta>1$, a small potential barrier for secondary electrons will be formed near the wall, to guarantee that the number of secondary electrons actually leaving the wall is equal to the difference between the number of incoming electrons and ions. The amplitude of this barrier will obviously be of the order of the "temperature" of the secondary electrons, i.e., a few electron-volts.

The most obvious solution to the problem of the secondary emission would be the use of a suppressor grid, and we discuss this possibility at the end of this section. On the other hand, although simple in principle, this techniques brings up an additional complexity to the overall design. This is why we consider first other possible solutions.

They are based on the observation that, in order to reach the region where the potential barrier for plasma electrons is formed, the secondary electrons would have to overcome a substantial mirror ratio, $\sim K_{m i r}\left(m_{e} / m_{i}\right)^{1 / 2}>>1$. Therfore, if secondary electrons acquire a non-negligible perpendicular velocity near the wall, they will be reflected back and absorbed. In this regard, the flatness of the potential distribution in the outlying part of the end tank is a favorable factor, as the electric field that pulls the electrons towards the mirror is weak, and the "Yushmanov potential" has a maximum even for quite a modest electron magnetic moment.

\section{IV.B. Two-stream instability}

One possible mechanism of electron scattering is related to the fact that the secondary electrons, when accelerated in the Debye sheath, form a beam (Fig. 3).
Such a distribution function is unstable with respect to a beam-plasma instability and one can expect that the beam will be scattered by the unstable Langmuir waves and then reflected back to the wall by the converging magnetic field. In this way, the problem of accumulation of the cold electrons would be eliminated.

The flux of the secondary electrons from the wall is $\sim n_{\text {mir }} u / K_{\text {wall }}$. Their density just beyond the Debye sheath is

$$
n_{\text {beam }} \sim n_{\text {mir }}\left(u / K_{\text {wall }} \hat{\mathrm{v}}\right) \sim n\left(\frac{m_{e}}{K_{\text {wall }} m_{i}}\right)^{1 / 3},
$$

whereas the plasma density near the wall is $n_{\text {mir }} / K_{\text {wall }}$. The growth rate corresponding to the scattered beam can be evaluated as

$$
\Gamma \sim \omega_{p e} \frac{n_{\text {beam }}}{n} \sim \omega_{p e}\left(\frac{m_{e}}{K_{\text {wall }} m_{i}}\right)^{1 / 3},
$$

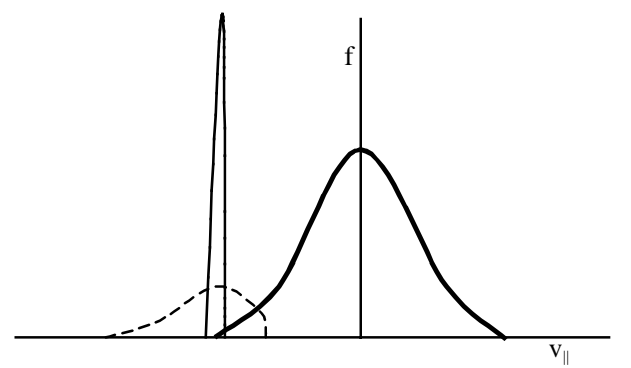

Fig. 3 Electron distribution function in the vicinity of the wall (but beyond the Debye radius). Shown in a bold line is a distribution function of the trapped electrons. A thin continuous (dashed) line depicts secondary electrons before (after) the relaxation took place. In the case when the electron gyrofrequency is of order of the electron plasma frequency, the relaxation is accompanied by a substantial angular scattering.

where $\omega_{p e}$ is the local plasma frequency. The group velocity of the Langmuir oscillations excited by the beam in a plasma with $\omega_{c e}>\omega_{p e}$ is of order of the beam velocity. This means that the length $L_{\text {inst }}$ required for the development of this instability is

$$
L_{i n s t} \sim 3 \frac{\hat{\mathrm{v}}}{\Gamma} \sim 3 \frac{\mathrm{v}_{T e}}{\omega_{P e}}\left(\frac{m_{i}}{m_{e}}\right)^{1 / 2}
$$

The factor 3 reflects the fact that a few e-folding lengths are needed for the unstable wave to grow to a nonnegligible level from the initial fluctuations. For the nearwall densities indicated in Table I, the length is $\sim 10 \mathrm{~m}$ for the GDT and $\sim 1000 \mathrm{~m}$ for the standard mirror, the latter meaning that the beam instability can hardly play any role for the standard mirror. Conversely, the length of $10 \mathrm{~m}$ derived for GDT is quite compatible with the dimensions of the end tank. One should also mention that, in case of a standard mirror, one has $\omega_{c e} / \omega_{p e}>1$ (see Table II), this meaning that the electrons cannot be scattered by the Langmuir waves because of the conservation of their 
magnetic moment. In the case of GDT, the two frequencies are comparable, making the scattering possible.

\section{IV.3. Tilting the end plate}

It might be beneficial to use, instead of the plate normal to the field lines (as shown on Fig. 1), a segmented plate of a "Venetian-blind" type [10] shown on Fig. 4. The segments form a very shallow angle $\alpha$ with a local magnetic field. This brings up two potentially favorable effects.

The first effect is related to the observation that, at a small $\alpha$, almost all secondary electrons return to the plate in the course of their gyro-motion. As these electrons have a small energy, they do not cause any secondary emission. Therefore, the secondary emission is strongly suppressed.

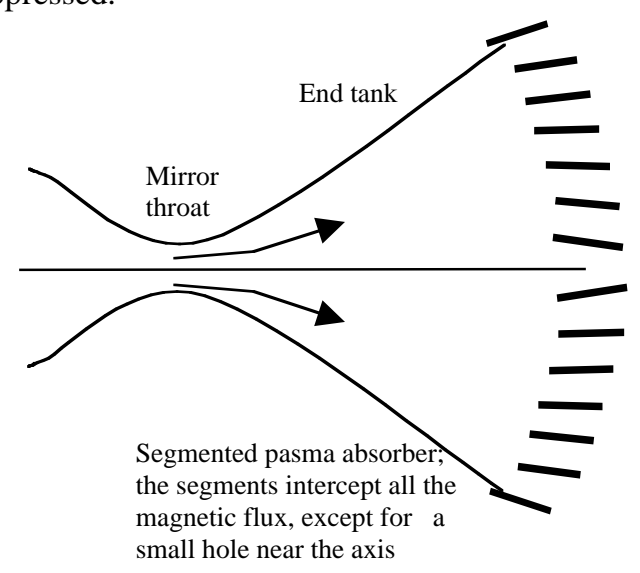

Fig. 4. A segmented plasma absorber

Complications may arise from the fact that there is a substantial electric field in the Debye sheath that pulls electrons away from the surface. The effect of this field on the electron trajectories has been studied in Ref. [11]; the possible role of the surface roughness was considered in Ref. [12]. In the case of a canonical mirror, where the electron gyro-radius is very small compared to the Debye radius, the role of the electric field is relatively small, although a more detailed analysis is needed, to see if the suppression of the secondary emission is sufficient. In the case of the GDT, where the gyroradius is only by a factor of 2 or 3 less than the Debye radius, the electric field certainly plays a strong role and the suppression of the secondary emission by the tilt of the plates does not work.

In the case of GDT, another beneficial effect comes into play. Because of the tilt, the secondary electrons that have initially a very small energy, acquire a substantial kick from the electric field which is, in the case of a strong tilt, directed almost perpendicularly to the surface. Because of that, the secondary electron starts its motion having a large transverse velocity (in the drift frame),

$$
\mathrm{v}_{\perp}=c \frac{E}{B_{\text {wall }}} \sim \hat{\mathrm{v}} \frac{\omega_{c e}}{\omega_{p e}} .
$$

In the case of GDT, where electron Debye radius is comparable to the electron gyroradius, this velocity is only a couple of times less that the parallel (to the magnetic field) velocity that electrons acquire after traversing the Debye sheath. So, when they move away from the plate, they are reflected back by the increasing magnetic field.

\section{4 Using a suppressor grid}

The most direct (although probably not the most elegant) solution to the problem of the secondary emission would be introducing a suppressor grid. To assess the feasibility of this approach, one has to consider two factors: the heat load to the grid, and the magnitude of the Debye radius.

As seen from Table I, the heat load on the grid is such that the grid can withstand it even with a passive (radiative) cooling: even at a heat load of $1 \mathrm{MW} / \mathrm{m}^{2}$ the temperature increment of the passively cooled grid will not exceed $1500 \mathrm{~K}$.

Assuming that the voltage applied to the suppressor grid with respect to the wall is $1000 \mathrm{~V}$, one finds that the effective gap (determined by the space-charge effect) can be made as high as $2.5 \mathrm{~cm}$ for the gas-dynamic trap, and $25 \mathrm{~cm}$ for the canonical mirror. In the latter case, the grid can have a rather coarse structure. One can even use an active water-cooling of the grid rods, as they can have quite a substantial diameter. Still, the suppressor grid of the scale required for mirror reactors is not a trivial structure from the engineering standpoint and alternative ways of suppressing the secondary emission look preferable.

\section{THE ROLE OF THE NEUTRAL GAS}

The plasma hitting the end wall recombines there and produces an influx of neutral atoms. Assuming that only $1 / 30$ of the whole surface of the tank is covered with cryopumps, and that the sticking coefficient is 0.1 , one finds that every neutral will have to make 300 transits through the tank, before it is removed from the system. For the tank with a diameter of $20 \mathrm{~m}$, this yields the distance of $\sim 6 \cdot 10^{5} \mathrm{~cm}$. On the other hand, the ionization mean free path is equal to

$$
l_{i} \sim \frac{\mathrm{v}_{a t}}{n_{\text {wall }}\langle\sigma \mathrm{v}\rangle_{i}},
$$

where $\mathrm{v}_{\mathrm{at}}$ is the characteristic velocity of the neutral atoms. Assuming that the ionization parameter $\langle\sigma v\rangle_{i}$ is $10^{-8} \mathrm{~cm}^{3} / \mathrm{s}$, and that the characteristic velocity of the neutrals is $3 \cdot 10^{5} \mathrm{~cm} / \mathrm{s}$, one finds that $l_{i}$ is $3 \cdot 10^{7} \mathrm{~cm}$ for the canonical mirror, and $3 \cdot 10^{5} \mathrm{~cm}$ for the GDT (see the second to the last column in Table 1). In other words, in 
the case of the "canonical" mirror, the neutrals are pumped out well before they are re-ionized, and do not contribute to the energy and particle balance in the system. Conversely, in the case of GDT a substantial fraction of the neutrals will be reionized. The cold electron population produced in this process will lower the potential barrier for the hot plasma electrons and thereby lead to an increase the electron heat loss. To avoid this outcome, one might consider increasing the number of cryopumps and the sticking coefficient.

The steady-state density of the neutral atoms evaluated for the aforementioned parameters of the system will be quite significant, $5 \cdot 10^{11} \mathrm{~cm}^{-3}$ for the canonical mirror, and $3 \cdot 10^{12}$ for GDT.

\section{NON-STEADY-STATE EFFECTS}

Thus far we have been considering a steady state operation of the mirror device. However, the collisional times that lead to establishing steady-state distribution functions in the end tank are quite long. As was mentioned in Sec. III, they are $6 \cdot 10^{3} \mathrm{~s}$ (!) for the "canonical" mirror and $5 \mathrm{~s}$ for the GDT. In other words, these are macroscopic times.

On the one hand, this points at the "fragility" of the distribution functions established in the expander tanks. Even minor perturbations of various types may cause the change of the equilibrium state. In particular, the ionization of the neutral gas may substantially modify the distribution function and lead to a confinement degradation.

On the other hand, especially in the case of a "canonical" mirror, where the characteristic times in the end tank are in the range of hours, one can consider a non-steady-state mode of operation, with the width of the pulses shorter than characteristic internal times of the endtank plasma. The whole issue of the role of neutrals and non-steady-state effects requires further work, before any firm predictions can be made.

\section{DISCUSSION}

In the case of a small secondary emission from the end plates (roughly, at $\eta<0.5$ ), electron heat losses are strongly inhibited by the electrostatic potential barrier in the end tank and are quite modest. They do not impose any insurmountable constraints on the reactor design.

Strong flaring of the magnetic field in the end tank required for reduction of the heat load on the end plate (plasma absorber) brings up an interesting effect: if the expansion ratio $K_{\text {wall }}$ exceeds, roughly $\left(m_{i} / m_{e}\right)^{1 / 2}$, the potential distribution in the outer part of the end tank, where the local expansion ratio $K$ is greater than $\left(m_{i} / m_{e}\right)^{1 / 2}$, the potential distribution flattens [5]. In this outer zone the majority of electrons are trapped electrons, with passing electrons (i.e., electrons originated in the mirror throat) being a tiny minority. The presence of this flat zone is very important for several techniques helping to reduce a detrimental effect of the secondary electrons released from the end plate.

The secondary emission from the end plate becomes of a concern when the secondary emission coefficient $\eta$ becomes greater than 0.5 .. A dramatic deterioration may happen if it approaches or exceeds unity. In this case, the initially cold secondary electrons may accumulate in the end tank and reduce the electrostatic potential barrier for the hot electrons, thereby causing a significant (10-fold) increase of the electron heat loss.

The most direct way of reducing the flux of the secondary electrons is the use of a suppressor grid. In the case of "standard" mirrors the grid can be quite coarse and even allows for a water cooling through the internal channels. For GDT, the grid must be much finer and has to withstand somewhat higher heat fluxes, that makes its development not a trivial engineering endeavor.

However, in the case of GDT there exist more attractive ways of suppressing the secondary emission. First, there may be no need in the use of any active suppression techniques, because the secondary electrons would excite the beam-plasma instability, will experience angular scattering and will be reflected back to the end plate. If this approach does not work, one can use a venetian-blind type structure, with a strong tilt of the individual plates with respect to the magnetic field. In this case, the secondary electrons get a strong kick in the transverse direction and then get reflected back to the end plate from the region of stronger magnetic field. Both these mechanisms rely heavily on the flatness of the electrostatic potential distribution in the large part of the end tank and the corresponding absence of a strong electric field that would pull the secondary electrons to the main plasma.

These two mechanisms are not very promising in application to the "standard" mirror, where, however, a different mechanism may work, associated with the return of the secondary electrons to the tilted plates (see Ref. [11]). Whether this mechanism actually works, needs to be explored more carefully.

Non-trivial effect may arise from the recycling of neutral atoms in the end tank. Electrons formed by the ionization of these neutrals may add to the undesirable effect of forming a cold neutralizing electrons. This effect needs a more detailed analysis.

It has to be kept in mind that radial plasma transport, entirely neglected in this paper, may contribute to the electron cooling, very much as it does in tokamaks. All in all, however, there is no reason to believe that the fast electron cooling, especially the axial cooling, is an inevitable feature of open confinement systems. 


\section{ACKNOWLEDGMENTS}

The author is grateful to Dr. I.A. Kotelnikov for presenting this paper at the Open Systems 2004 Conference. Work performed under the auspices of the U.S. Department of Energy by University of California Lawrence Livermore National Laboratory under contract No.W-7405-Eng-48.

\section{REFERENCES}

[1] D.E. Baldwin. "End-loss processes from mirror machines." Rev. Mod. Phys., 49, 317 (1977).

[2] V.P. Pastukhov. "Classical longitudinal plasma losses from open adiabatic traps." In: Reviews of Plasma Physics, v. 13, p. 203, B.B. Kadomtsev, Ed. (Consultants Bureau, NY, 1987).

[3] D.D. Ryutov. ."Open-ended traps." Soviet Physics Uspekhi, 31, 300 (1988).

[4] V.V. Mirnov, D.D. Ryutov. "Gas Dynamic Trap," In: "Summaries in Science and Technology - Plasma Physics”, V.D. Shafranov, Ed., v. 8, p. 77, Moscow, VINITI Publ. House, 1988 (in Russian).

[5] I.K. Konkashbaev, I.S. Landman, F.R. Ulinich. "Possibility of decreasing the electron heat flux from open traps." Sov. Phys. JETP, 47, 501 (1978).

[6] S.I. Braginski. "Transport processes in a plasma." In: Reviews of Plasma Physics, v. 1, p. 205 M.A. Leontovich, Ed. (Consultants Bureau, NY, 1965).

[7] R.F. Post . "The magnetic mirror approach to fusion." Nucl. Fusion, 27, 1579 (1987).

[8] V.V. Mirnov, D.D. Ryutov. "Linear gasdynamic system for plasma confinement". Sov. Tech. Phys Lett., 5, p.279 (1979)

[9] G.D. Hobbs, J.A. Wesson. "Heat flow through a Langmuir sheath in the presence of electron emission." Plasma Physics, 9, 85 (1967).

[10] R.W. Moir, W.L. Barr. "venetian-blind direct energy converters for fusion reactors." Nuclear Fusion, 13, 35 (1973).

[11] S. Mizoshita, K. Shiraishi, N. Ohno, S. Takamura, "Secondary electron emission from solid surface in an oblique magnetic field" J. Nucl. Mater., 220-222, 488 (1995).

[12] R.H. Cohen, D. D. Ryutov. "Plasma near a divertor plate: effects of surface roughness and particle drifts." Contrib. Plasma Phys., 40, 456 (2000). 